\title{
Chalenges and Opportunities in Mathematical and Theoretical Biology and Medicine: Foreword to Volume 2 (2013) of Biomath
}

The challenges faced today at the interface of the computational, life, social, mathematical and data sciences expand as the world's population approaches the seven billion mark. Today, we are asked to address questions over multiple temporal and spatial scales as well as across various levels of organization. In the process, the world has placed mathematical scientists, broadly defined to include computational, computer, statistics and data science experts, at the heart of trans-disciplinary and interdisciplinary efforts aimed primarily at assessing arguably the most important technical challenge of our times, namely, how to model and quantify uncertainty. Twenty first century research is driven to a great extent by questions involving the study of the dynamics of biological process. And so, we have been forced to account for the impact of social systems, bringing the role of human-decisions to the forefront of most efforts to put the power of the quantitative sciences into the hands of policy and decision makers.

The challenges faced by mathematical scientist include the development and implementation of frameworks and methods aimed at assessing and mitigating the impact of health disparities; the identification, understanding and management of sustainability concepts and systems; the development and systematic implementation of holistic approaches, a key to the study of the dynamics and control of biological systems; the development of methods and approaches that help evaluate uncertainty in current studies of climate change, including global warming; the need to find scalable solutions that address issues of food security and world hunger; and more.

A prototypical and timely global challenge, that cuts across issues of sustainability, evolution and disease dynamics, is briefly discussed in this introduction as a way of highlighting the challenges posed by the study of phenomena across multiple temporal and spatial scales and levels of organization. On March 11, 2013, the British Chief Medical Officer Dame Sally Davies The Independent, Tuesday 12 March 2013 observed that "the problem of microbes becoming increasingly resistant to the most powerful drugs should be ranked alongside terrorism and climate change on the list of critical risks to the nation. ... Yet while antibiotic use is rising not least in agriculture for farmed animals and fish ... resistance is steadily growing and the pipeline is drying up ... of new drugs which can replace those becoming useless. [In fact] No new classes of antibiotics have been developed since 1987, and none are in the pipeline." Professor Nigel Brown, president of the Society for General Microbiology remarks that immediate action by scientists is required if we are going to identify and mass produce new antibiotics; the kind of effort needed to tackle the problem of antimicrobial resistance and its transmission, particularly in the context of nosocomial (hospital) infections. (Ibid.)

The head of the WHO while addressing a meeting of infectious disease experts in Copenhagen, highlighted the global crisis in antibiotics, the result of "rapidly evolving resistance among microbes responsible for common infections that threaten to turn them into untreatable diseases ... every antibiotic ever developed was at risk of 
becoming useless. A post-antibiotic era means, in effect, an end to modern medicine as we know it. Things as common as strep throat or a child's scratched knee could once again kill ... Antimicrobial resistance is on the rise in Europe, and elsewhere in the world. We are losing our first-line antimicrobials." (The Independent, Friday 16 March 2012). The issues of the persistence, evolution and the expansion of resistance to antimicrobials are important because the number of drugs available is limited with no new ones being developed for the past three decades. The magnitude of the catastrophes predicted by the British Chief Medical Officer Dame Sally Davies and by the Director General of the World Health Organization, Dr. Margaret Chan on the global challenges posed by antibiotic resistance, must therefore be assessed and monitored and mathematical scientists have the tool kits needed to make a difference.

My own experiences as a past participant, in a variety of roles nearly three decades ago, in the schools of ecology organized at the International Centre for Theoretical Physics (ICTP), Trieste Italy, led for several years by Tom Hallam, Lou Gross and Simon Levin, brought me head on for the first time in my life with the most important resource that we have to address the grand global challenges that dominate the twenty-first century, namely, the talent, energy, passion, foresight and commitment of young researchers across the world, an experience repeated in 2013 in Sofia, Bulgaria.

The series BIOMATH conferences carried, unfortunately not continuously, for over a couple of decades, provide the international environment needed for mentoring and promoting the career of young individuals, talented mathematical scientists, who are passionate about issues of national and international impact. BIOMATH conferences are "devoted to recent research in biosciences based on applications of mathematics as well as mathematics applied to or motivated by biological applications." Its commitment to building multi-national environments that foster interdisciplinary and trans-disciplinary research is expressed explicitly in its chart that identifies this venue as "a multidisciplinary meeting forum for researchers who develop and apply mathematical and computational tools to the study of phenomena in the broad fields of biology, ecology, medicine, biotechnology, bioengineering, environmental science, etc."

This volume includes selected papers from the presentations at the 2013 BIOMATH Conference. They provide a sample of the richness, talent and diversity of the community of mathematical scientists that participated in this event. The articles involve applications to cell biology, epidemiology, immunology and ecology as well as methodological work in numerical methods, optimal control, statistics and network theory.

I was proud to be asked to participate at the 2013 BIOMATH conference held in Sofia, Bulgaria, honored to be asked to write a foreword for Volume 2 of Journal BIOMATH, blessed by the opportunity to listen and learn from talented individuals and develop new friendships.

\section{Carlos Castillo-Chavez}

Arizona State University

Math., Comp., and Model. Sciences Ctr.

Tempe, AZ, USA

Email: ccchavez@asu.edu 